\title{
Isolated Handwritten Digit Recognition using Adaptive Unsupervised Incremental Learning Technique
}

\author{
Dharamveer Sharma \\ Assistant Professor, \\ Department of Computer Science, Punjabi \\ University, Patiala
}

\author{
Deepika Gupta \\ MTech (Computer Science), \\ Department of Computer Science, Punjabi \\ University, Patiala
}

\begin{abstract}
This paper presents a new approach to off-line handwritten numeral recognition. From the concept of perturbation due to writing habits and instruments, we propose a recognition method which is able to account for a variety of distortions due to eccentric handwriting. The recognition of handwritten numerals is a challenging task in the field of image processing and pattern recognition. It can be considered as one of the benchmarks in evaluating feature extraction methods and the performance of classifiers. The performance of character recognition system depends heavily on what kind of features are being used. The objective of this paper is to provide efficient and reliable techniques for recognition of handwritten numerals. In this paper we propose Zoning based feature extraction system which calculates the densities of object pixels in each zone. Firstly the whole image is divided into $4 \times$ 4 zones. Further in order to gain more accuracy these zones are divided into $6 \times 6$ zones. The division of zones carried out up to $8 \times 8$ zones. Hence 116 features are extracted in all. Nearest neighbour classifier is used for subsequent classification and recognition purpose.
\end{abstract}

\section{INTRODUCTION}

Optical character recognition, usually abbreviated to OCR is the mechanical or electronic translation of images of handwritten, typewritten or printed text (usually captured by a scanner) into machine-editable text or computer process-able format, such as ASCII code. Whenever a page is scanned, it is stored as a bit-mapped file. When the image is displayed on the screen, we can read it. But it is just a series of dots for the computer. The computer does not recognize any "words" on the image. OCR makes the computer read these words. It looks at each line of the image and determines which particular character is represented by dots.

OCR is a field of research in pattern recognition, artificial intelligence and machine vision. Optical character recognition (using optical techniques such as mirrors and lenses) and digital character recognition (using scanners and computer algorithms) were originally considered separate fields. Because very few applications survive that use true optical techniques, the OCR term has now been broadened to include digital image processing as well.

The recognition of handwritten characters by computer has been a topic of intensive search for many years. Handwritten numeral recognition is always the research focus in the field of image process and pattern recognition. The numeral varieties in size, shape, slant and the writing style make the research harder. The numeral character recognition is the most challenging field, because the big research and development effort that has gone into it has not solved all commercial and intellectual problems. Handwritten numeral character recognition is an important step in many document processing applications. Digital document processing is gaining popularity for application to office and library automation, bank and postal services, publishing houses and communication technology. The complexity of the problem is greatly increased by noise in the data and infinite variability of handwriting as a result of mood of writer and nature of writing. Recognition of handwritten digits has been popular topic of research for many years. The recognition of handwritten numeral character has been considerable interest to researchers working on OCR.

For the testing purpose an Award List has been used. Data has been collected from 200 users and extracted individual digits from these forms. These forms are filled out from different users in order to take different samples of handwriting. This Award List has 4-digit Roll no, 4-digit Code no and 3-digit Marks. The outcome of the research will be an automated system for recognition of awards. This automated system will recognize only digits. This procedure can also be done manually, but that is a tedious task and prone to error. This system's complexity lies in the different handwriting styles which vary from human to human. Thus automated system provides better recognition accuracy than manual system. The award list which is used in the automated system is shown below:

The rest of the paper is organized into five sections. In the Section 2 we will briefly explain about the review of literature in which the feature extraction technique along with the classifier is discussed. Section 3 describes the proposed system. In section 4 we will discuss about Recognition Result and Comparisons among Different Zoning Techniques and finally conclusion is given in section 5 .

\section{REVIEW OF LITERATURE}

The recognition of handwritten numerals is a challenging task in pattern recognition. It can be considered as one of the benchmarks in evaluating feature extraction methods and the performance of classifiers. P.Zhang, T.D.Bui, C.Y.Suen [1] have proposed a new character recognition algorithm using MAT based Directional Features, Binary Gradient Directional Features, and, Image Thinning Distance Feature.

M. Ziaratban, K. Faez, F. Faradji [2] presents an innovative approach for character recognition called Template Matching. This technique extracts features by searching the special templates in input images. For each template, the position of the best matching in an image is found and saved. The amounts of matching can also be used as a feature.

Hu and Yan [3] presented a structural method for describing both printed and handwritten characters. The character is decomposed into primitives, and each primitive is described by a primitive code. The topological information of a character is represented by a global code. The global code and the primitive 
codes describe each character clearly and effectively. Heutte [4], present a new feature vector for the recognition of handwritten characters which combines the strengths of both statistical and structural feature extractors and provides a wide range of identification clues.

Y. Hamamoto, S. Tomita, M. Koga and H. Fujisawa [6, 7, 8] presents a 2D Gabor filter which is a band-pass spatial filter and selective to both orientation and spatial frequency. A Gabor filters extract the orientation-dependent frequency contents, i.e. edge like features, from as small an area as possible.

F. Kimura and U. Pal [9] have proposed a new character recognition algorithm using Gradient filter which are applicable to gray-scale images to obtain a normalized image, and are immune to image noise. A Roberts filter is applied on the normalized image to obtain gradient image.

DDD (Directional Distance Distribution), proposed by Oh and Suen [10], is based on the distance information computed for both black pixels and white pixels in 8 directions. Structural representation may also encode some knowledge about the shape and structure of the character or about various components that form the character. Some feature which could check number of horizontal and vertical line, end points, presence of loops, number of loops, position of loop, and number of intersections and junctions.

The performance of a character recognition system depends on what kinds of features are being used to recognize handwritten numerals. Selection of a feature extraction method is probably the single most important factor in achieving high recognition. Since we have been used Zoning feature extraction method for recognition of handwritten numerals. Zoning is a Distance metric based feature extraction system [5].

In the first type of zoning method features are extracted for each zone by dividing the whole image into equal number of zones. The number of zones is fixed in this method and they are $6 \times 6$. The densities of object pixels in each zone are calculated. Density is calculated by finding the number of object pixels in each zone and dividing it by total number of pixels. Densities are used to form a representation. For binary images, a value of each pixel is either 1 or 0 . We have considered pixels having value BLACK $(0)$ as object pixel.

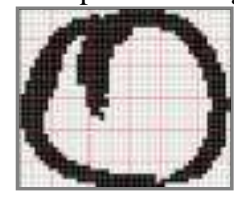

(a)

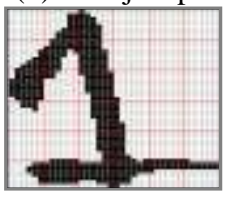

(b)
Figure 1: Original Matrix for Numeric Digits 0(a) and 1(b) In the next zoning method a frame containing the numeral whose centroid is computed and then image is divided into $6 \times$ 6 equal zones. Compute the distance between the image centroid to each pixel present in the zone using Euclidean Distance method. Then compute the average distance between these points. This procedure is sequentially for the entire zone present in the numeral image.

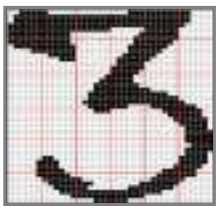

(a)

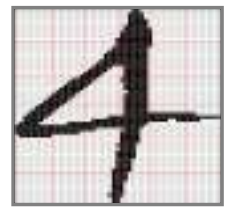

(b)
Figure 2: Original Matrix for Numeric Digits 3(a) and 4(b) These zoning methods can also be combined in order to gain more recognition accuracy. Finally the extracted features from the feature extraction stage are passed to classification stage to identify the text segment. The objective of the classifier is to provide efficient and reliable techniques for recognition of unconstrained handwritten numerals. The classifier is used to make a final decision according to extracted features. The classifier is used to solve the complex problem of digit recognition. The classification phase uses the features extracted from the previous stage to identify the text segment according to preset rules.

Neocognitron is a multi-layered neural network with a property of being able to recognize patterns with deformation, changes in size or shift in position [11, 12,13,]. There are pairs of layers, S-layers with S-cells and C-layers with C-cells. S-cells receive their inputs from previous $\mathrm{C}$-layer except for the first layer which is connected to the input image. . S-cells in the same S-plane extract the same feature at slightly different location to allow for positional shift tolerance in the features represented by these S-cells. The whole recognition process is conducted from layer to layer where local feature extracted in the lower layers are passed onto upper layers for more complex feature extraction until one of the C-cells in the final C-layer produces the response to the input pattern.

SVM (Support Vector Machine) is a useful technique for data classification [15, 16, 17]. The Support Vector Machine (SVM) is learning machine with very good generalization ability, which has been applied widely in pattern recognition, regression estimation, isolated handwritten character recognition, object recognition speaker identification, face detection in images and text categorization. SVM implements the Structural Risk Minimization Principal which seeks to minimize an upper bound of the generalization error rather than minimize the training error.

The best known classifier is KNN (K- Nearest Neighbor). It does not require prior information about the data. An incoming pattern is classified using the cluster, whose center is the minimum distance from the pattern over all the clusters. The task here is to design a model using training data which can classify the unknown patterns based on that model. For the training purpose, we have used 10000 numeric digits. Firstly feature vector for all training data is produced and stored in files.

\section{PROPOSED SYSTEM}

For extracting the feature, the zone based feature extraction method is purposed. The most important aspect of handwriting recognition scheme is the selection of good feature set, which is reasonably invariant with respect to shape variations caused by various writing styles. Zone based feature extraction method provides good result even when certain preprocessing steps like filtering, smoothing and slant removing are not considered. In this section, we explain the concept of feature extraction method used for extracting features for efficient classification and recognition.

Before extracting features using feature extraction method segmentation is performed on the training form for extract isolated handwritten numeral images. Segmentation is an operation that seeks to decompose an image of sequence of characters into sub images of individual symbols. Segmentation is the first and most important step towards automated recognition of any handwritten or printed data. Here 
in the training phase training form has been used; from which isolated handwritten numeric digits, using horizontal and vertical projection are extracted. Features are extracted using reference points, which diagnose from where to start extracting features. It starts diagnosing from top left reference point to bottom right reference point to extract isolated numeric digits. For extracting features it first creates horizontal profiles after these vertical profiles are created on horizontal profiles.

For the training purpose sample form has been used. 200 forms for sample and image data collection has been used. 200 training sets of such images, each one containing 50 characters are used for training. Each set of character images varies for handwriting styles. The training sets of characters are shown in tables 3.1 to 3.10. Adaptive Unsupervised Incremental Learning Technique has been used for Training purpose. A file database has been created which contains isolated images of handwritten numeric digits which are collected using a form containing 5-sets of all the 10 digits of decimal number set. Each user will fill these set of forms in his/her own handwriting. The following paragraph explains in detail about the feature extraction methodology. The sample form for collecting data is as below.

\section{Sample Form for Training:}

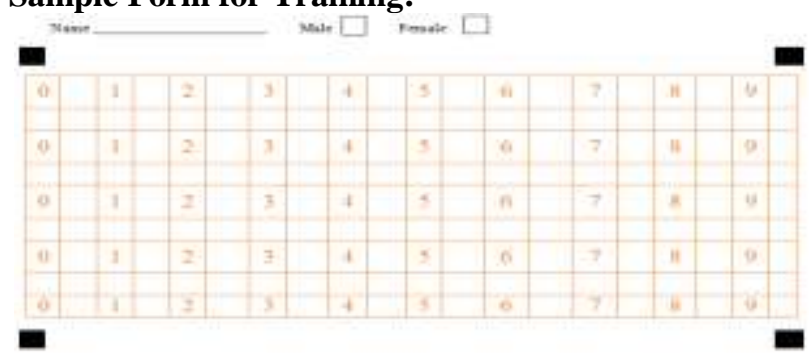

Figure 3: Sample data collection form

The sample of collected data is:

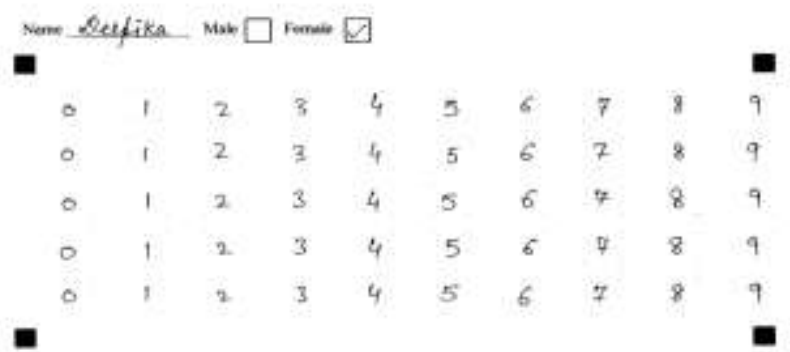

Figure 4: Example of a sample form with collected data Zone based feature extraction method is purposed in this paper. In this zoning method again features are extracted for each zone by dividing the whole image into number of zones, but here the numbers of zones are not fixed. Firstly the whole image is divided into $4 \times 4$ zones. Further in order to gain more accuracy these zones are divided into $6 \times 6$ zones. The division of zones carried out up to $8 \times 8$ zones. Again the density is calculated by finding the number of object pixels in each zone and dividing it by total number of pixels.

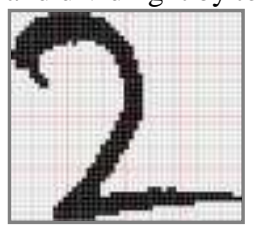

(a)

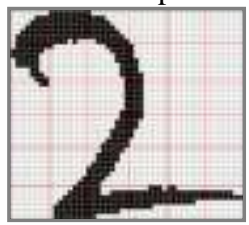

(b)

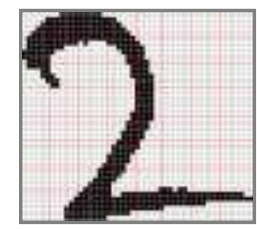

(c)
Figure 5: Original Matrices for Numeric Digit (2)
This procedure is sequentially for the entire zone present in the numeral image. Finally 116 such features are extracted for classification and recognition. For classification and recognition NNC classifier is used.

Offline isolated handwritten image samples are extracted using zoning feature extraction method. These are the original image drawn by user by free handwriting that stores in a file databases. This file database makes an image model library in which different types of binary images drawn by different users using different styles of handwriting are stored. The following are the image samples of Numeric characters:-

Table 1: Sample of Offline Handwritten Digits Images 0-9

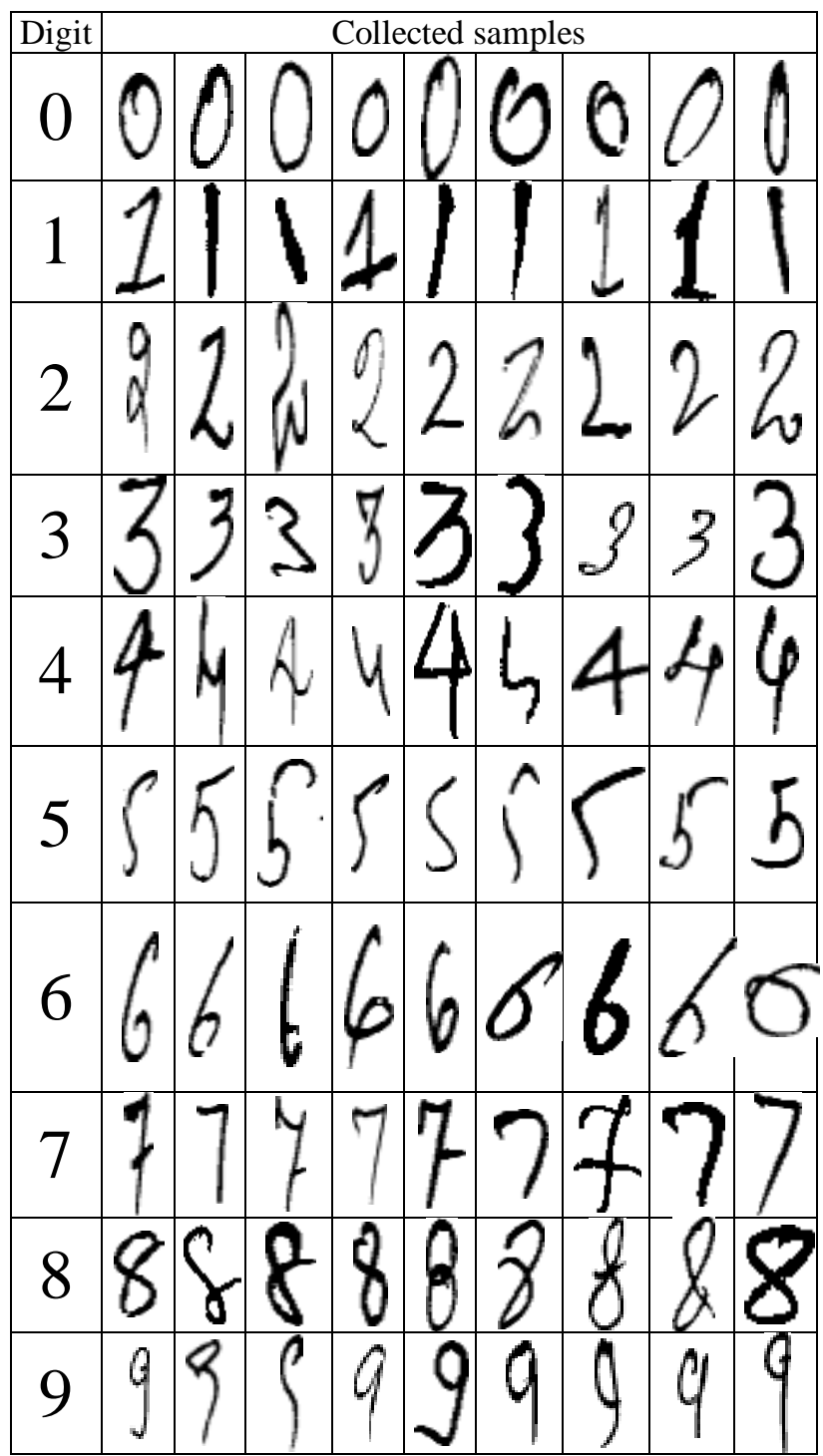

The final stage is testing phase which actually recognize the isolated handwritten numeric images using the $\mathrm{k}$-nearest neighbor classifier with the value of $\mathrm{k}=1$. For the testing purpose an Award List has been used, which contains 4-digit Roll no, 4-digit Code no and 3-digit marks which is as below: 


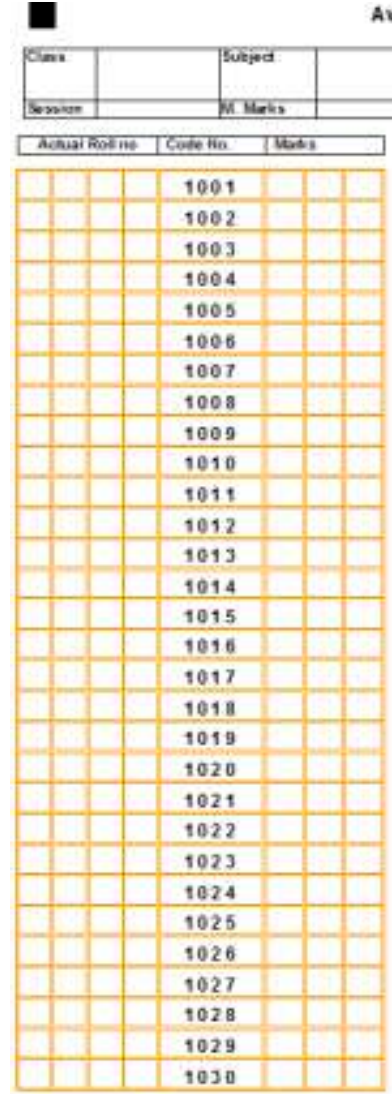

Pax
Award List
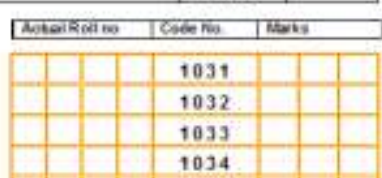

103

1036

1037

1038

1039

1041

104

1043

1044

1045

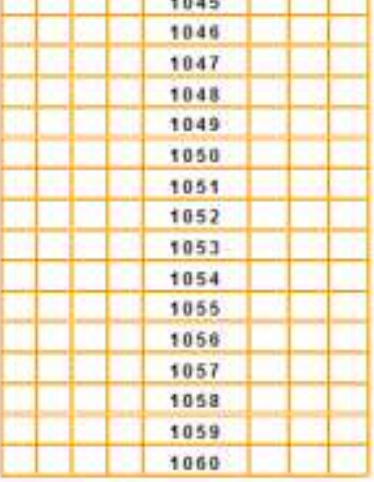

Pas $\square$ Fail

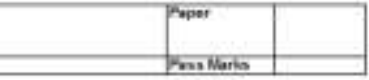

Figure 6: A Sample award list form

Segmentation is a kind of phase which has been adopted in both the training and testing phase. Segmentation is an operation that seeks to decompose an image of sequence of characters into sub images of individual symbols. Character segmentation is a key requirement that determines the utility of conventional systems. Different methods used can be classified based on the type of text and strategy being followed like straight segmentation method, recognition-based segmentation and cut classification method.

Segmentation is the first and most important step towards automated recognition of any handwritten or printed data. Classification is performed on these segmented characters without further interaction with the segmentation process. Dissection is an intelligent process in which analysis of the image is carried out.

Horizontal Projection: For a given binary image of size $\mathrm{M} \times$ $\mathrm{N}$, where $\mathrm{M}$ is the height and $\mathrm{N}$ is the width, the horizontal projection is defined as:

$$
H P(i), i=1,2,3 \ldots M \text {. }
$$

Where $H P(i)$ is the total number of black (object) pixels in the $\mathrm{i}_{\mathrm{th}}$ horizontal row.

Vertical Projection: For a given binary image of size $\mathrm{M} \times \mathrm{N}$, where $\mathrm{M}$ is the height and $\mathrm{N}$ is the width, the vertical projection is defined as:

$$
V P(j), j=1,2,3 \ldots M .
$$

Where $V P(j)$ is the total number of black (object) pixels in the $\mathrm{j}_{\text {th }}$ horizontal row.

Zoning feature extraction algorithm along with the k-nearest neighbor classifier has been adopted, which provides the best recognition accuracy rate. The best recognition rate is provided by first type of zoning feature extraction algorithm with the value of $\mathrm{k}=1$.

The KNN classifier is an extension of one nearest neighbor $(1 \mathrm{NN})$ classifier. The $\mathrm{K}$ nearest neighbor $(\mathrm{KNN})$ method is a classification method and the principle based on the best distance measurement. It only needs reference data points for both classes $[14,15]$. A test sample is then attributed the same class label as the label of the majority of its $\mathrm{K}$ nearest (reference) neighbors. We calculate the Euclidean distance between the test point and all the reference points in order to find $\mathrm{K}$ nearest neighbors, and then rank the obtained distances in ascending order and take the reference points corresponding to the $\mathrm{k}$ smallest Euclidean distances. The k-nearest 30eighbor approach attempts to compute a classification function examining the labeled training points as nodes or anchor points in the $\mathrm{n}$-dimensional space, where $\mathrm{n}$ is the feature vector size. Rather than using a 1-nearest 30eighbor classifier, we choose a $\mathrm{k}-\mathrm{NN}$ classifier to reduce the effect of mislabeled training data and to get a better estimate of Input feature vector. Euclidean distance, used in k-NN finding the nearest 30eighbor, is the straight line distance between two points in an n-dimensional space. The Euclidean Distance between an input feature $\mathrm{X}$ and a library feature vector $\mathrm{C}$ is given by following equation:

$\mathbf{D}=\sqrt{\sum_{i}^{\mathrm{N}}}\left(\mathrm{c}_{\mathrm{i}}-\mathrm{X}_{\mathrm{i}}\right)\left(\mathrm{c}_{\mathrm{i}}-\mathrm{x}_{\mathrm{i}}\right)$

Where $c_{i}$ is the $i_{\text {th }}$ library feature and $x_{i}$ is the $i_{\text {th }}$ input feature and $\mathrm{N}$ is the number of feature used for classification. The class of library feature vector producing the smallest Euclidean Distance, when compared with the library input feature vector, is assigned to the input character. The $\mathrm{k}-\mathrm{NN}$ is more general than nearest neighbor. Putting it other way, nearest neighbor is a special case of $\mathrm{k}-\mathrm{NN}$, where $\mathrm{k}=1$. For the testing purpose the value of $k=1$ has been selected.

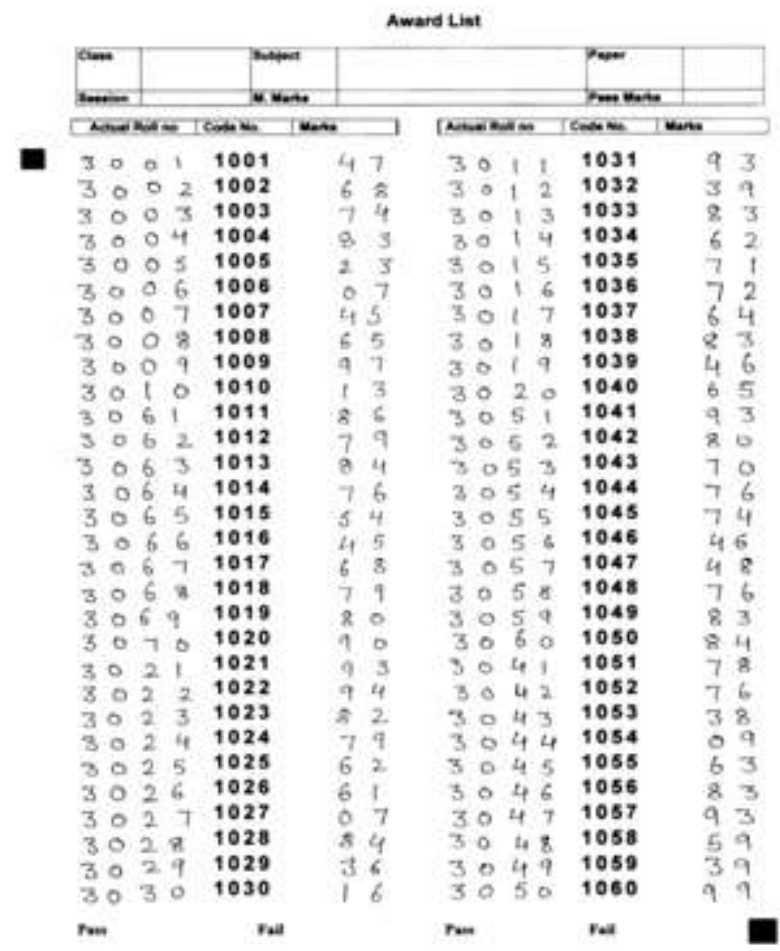

Figure 7: A filled award list form 
Table 2: Ground Truth Table for Form given in figure 7

\begin{tabular}{|l|l|l|l|l|l|l|l|l|l|}
\hline \multicolumn{7}{|c|}{ Actual Roll No. } & \multicolumn{1}{l|}{ Code No. } & \multicolumn{2}{l|}{ Marks } \\
\hline 3 & 0 & 0 & 1 & 1 & 0 & 0 & 1 & 4 & 7 \\
\hline 3 & 0 & 0 & 2 & 1 & 0 & 0 & 2 & 6 & 8 \\
\hline 3 & 0 & 0 & 3 & 1 & 0 & 0 & 3 & 7 & 4 \\
\hline 3 & 0 & 0 & 4 & 1 & 0 & 0 & 4 & 8 & 3 \\
\hline 3 & 0 & 0 & 5 & 1 & 0 & 0 & 5 & 2 & 3 \\
\hline 3 & 0 & 0 & 6 & 1 & 0 & 0 & 6 & 0 & 7 \\
\hline 3 & 0 & 0 & 7 & 1 & 0 & 0 & 7 & 4 & 5 \\
\hline 3 & 0 & 0 & 8 & 1 & 0 & 0 & 8 & 6 & 5 \\
\hline 3 & 0 & 0 & 9 & 1 & 0 & 0 & 9 & 9 & 7 \\
\hline 3 & 0 & 1 & 0 & 1 & 0 & 1 & 0 & 1 & 3 \\
\hline 3 & 0 & 6 & 1 & 1 & 0 & 1 & 1 & 8 & 6 \\
\hline 3 & 0 & 6 & 2 & 1 & 0 & 1 & 2 & 7 & 9 \\
\hline 3 & 0 & 6 & 3 & 1 & 0 & 1 & 3 & 8 & 4 \\
\hline 3 & 0 & 6 & 4 & 1 & 0 & 1 & 4 & 7 & 6 \\
\hline 3 & 0 & 6 & 5 & 1 & 0 & 1 & 5 & 5 & 4 \\
\hline 3 & 0 & 6 & 6 & 1 & 0 & 1 & 6 & 4 & 5 \\
\hline 3 & 0 & 6 & 7 & 1 & 0 & 1 & 7 & 6 & 8 \\
\hline 3 & 0 & 6 & 8 & 1 & 0 & 1 & 8 & 7 & 9 \\
\hline 3 & 0 & 6 & 9 & 1 & 0 & 1 & 9 & 8 & 0 \\
\hline 3 & 0 & 7 & 0 & 1 & 0 & 2 & 0 & 9 & 0 \\
\hline 3 & 0 & 2 & 1 & 1 & 0 & 2 & 1 & 9 & 3 \\
\hline 3 & 0 & 2 & 2 & 1 & 0 & 2 & 2 & 9 & 4 \\
\hline 3 & 0 & 2 & 3 & 1 & 0 & 2 & 3 & 8 & 2 \\
\hline 3 & 0 & 2 & 4 & 1 & 0 & 2 & 4 & 7 & 9 \\
\hline 3 & 0 & 2 & 5 & 1 & 0 & 2 & 5 & 6 & 2 \\
\hline 3 & 0 & 2 & 6 & 1 & 0 & 2 & 6 & 6 & 1 \\
\hline 3 & 0 & 2 & 7 & 1 & 0 & 2 & 7 & 0 & 7 \\
\hline 3 & 0 & 2 & 8 & 1 & 0 & 2 & 8 & 8 & 4 \\
\hline 3 & 0 & 2 & 9 & 1 & 0 & 2 & 9 & 3 & 6 \\
\hline 3 & 0 & 3 & 0 & 1 & 0 & 3 & 0 & 1 & 6 \\
\hline 3 & 0 & 1 & 1 & 1 & 0 & 3 & 1 & 9 & 3 \\
\hline 3 & 0 & 1 & 2 & 1 & 0 & 3 & 2 & 3 & 9 \\
\hline 3 & 0 & 1 & 3 & 1 & 0 & 3 & 3 & 8 & 3 \\
\hline 3 & 0 & 1 & 4 & 1 & 0 & 3 & 4 & 6 & 2 \\
\hline 3 & 0 & 1 & 5 & 1 & 0 & 3 & 5 & 7 & 1 \\
\hline 3 & 0 & 1 & 6 & 1 & 0 & 3 & 6 & 7 & 2 \\
\hline 3 & 0 & 1 & 7 & 1 & 0 & 3 & 7 & 6 & 4 \\
\hline 3 & 0 & 1 & 8 & 1 & 0 & 3 & 8 & 8 & 3 \\
\hline 3 & 0 & 1 & 9 & 1 & 0 & 3 & 9 & 4 & 6 \\
\hline 3 & 0 & 2 & 0 & 1 & 0 & 4 & 0 & 6 & 5 \\
\hline 3 & 0 & 5 & 1 & 1 & 0 & 4 & 1 & 9 & 3 \\
\hline 3 & 0 & 5 & 2 & 1 & 0 & 4 & 2 & 8 & 0 \\
\hline 3 & 0 & 5 & 3 & 1 & 0 & 4 & 3 & 7 & 0 \\
\hline 3 & 0 & 5 & 4 & 1 & 0 & 4 & 4 & 7 & 6 \\
\hline 3 & 0 & 5 & 5 & 1 & 0 & 4 & 5 & 7 & 4 \\
\hline 3 & 0 & 5 & 6 & 1 & 0 & 4 & 6 & 4 & 6 \\
\hline 3 & 0 & 5 & 7 & 1 & 0 & 4 & 7 & 4 & 8 \\
\hline 3 & 0 & 5 & 8 & 1 & 0 & 4 & 8 & 7 & 6 \\
\hline 3 & 0 & 5 & 9 & 1 & 0 & 4 & 9 & 8 & 3 \\
\hline 3 & 0 & 6 & 0 & 1 & 0 & 5 & 0 & 8 & 4 \\
\hline 3 & 0 & 4 & 1 & 1 & 0 & 5 & 1 & 7 & 8 \\
\hline 3 & 0 & 4 & 2 & 1 & 0 & 5 & 2 & 7 & 6 \\
\hline 3 & 0 & 4 & 3 & 1 & 0 & 5 & 3 & 3 & 8 \\
\hline 3 & 0 & 4 & 4 & 1 & 0 & 5 & 4 & 0 & 9 \\
\hline 3 & 0 & 4 & 5 & 1 & 0 & 5 & 5 & 6 & 3 \\
\hline
\end{tabular}

\begin{tabular}{|l|l|l|l|l|l|l|l|l|l|}
\hline 3 & 0 & 4 & 6 & 1 & 0 & 5 & 6 & 8 & 3 \\
\hline 3 & 0 & 4 & 7 & 1 & 0 & 5 & 7 & 9 & 3 \\
\hline 3 & 0 & 4 & 8 & 1 & 0 & 5 & 8 & 5 & 9 \\
\hline 3 & 0 & 4 & 9 & 1 & 0 & 5 & 9 & 3 & 9 \\
\hline 3 & 0 & 5 & 0 & 1 & 0 & 6 & 0 & 9 & 9 \\
\hline
\end{tabular}

\section{RECOGNITION RESULT AND COMAPRISONS AMONG DIFFERENT ZONING TECHNIQUES:}

We have experimented the system on handwritten numerals. The system is analyzed using different zoning feature extraction methods discussed in section 2 and 3 . The percentage accuracy is given in table 11. The percentage accuracy is calculated by dividing correctly recognized digits by total number of digits which are actually present.

Confusion Matrix: Confusion Matrix provides an easy and complete way to describe the knowledge about a classification result. The confusion matrix structure depends on the classifier performance and on consistence of utilized test set. The results of your confusion matrix highly depend on the selection of ground truth / test set pixels. Rows correspond to classes in the ground truth map (or test set). Columns correspond to classes in the classification result. The diagonal elements in the matrix represent the number of correctly classified pixels of each class, i.e. the number of ground truth pixels with a certain class name that actually obtained the same class name during classification. The off-diagonal elements represent misclassified pixels or the classification errors, i.e. the number of ground truth pixels that ended up in another class during classification.

Table 3: Confusion Matrix with K=1 (Zoning1)

\begin{tabular}{|c|c|c|c|c|c|c|c|c|c|c|}
\hline & 0 & 1 & 2 & 3 & 4 & 5 & 6 & 7 & 8 & 9 \\
\hline 0 & 1251 & 0 & 0 & 0 & 0 & 0 & 0 & 0 & 0 & 0 \\
\hline 1 & 0 & 1088 & 0 & 0 & 1 & 0 & 0 & 0 & 0 & 0 \\
\hline 2 & 0 & 0 & 426 & 0 & 0 & 0 & 0 & 0 & 0 & 0 \\
\hline 3 & 0 & 0 & 0 & 496 & 0 & 0 & 0 & 0 & 0 & 0 \\
\hline 4 & 0 & 0 & 0 & 0 & 471 & 0 & 0 & 0 & 1 & 0 \\
\hline 5 & 0 & 0 & 0 & 1 & 0 & 471 & 0 & 0 & 0 & 0 \\
\hline 6 & 0 & 1 & 0 & 0 & 0 & 0 & 269 & 0 & 0 & 0 \\
\hline 7 & 0 & 1 & 0 & 0 & 0 & 0 & 0 & 286 & 0 & 0 \\
\hline 8 & 0 & 0 & 0 & 0 & 0 & 0 & 0 & 0 & 302 & 0 \\
\hline 9 & 0 & 0 & 0 & 0 & 0 & 0 & 1 & 0 & 0 & 334 \\
\hline
\end{tabular}

Table 4: Digit wise recognition accuracy with $k=1$ (Zoning1)

\begin{tabular}{|r|r|r|r|}
\hline Digit & Total & Correct & Percentage \\
\hline 0 & 1251 & 1251 & 100 \\
\hline 1 & 1089 & 1088 & 99.91 \\
\hline 2 & 426 & 426 & 100 \\
\hline 3 & 496 & 496 & 100 \\
\hline 4 & 472 & 471 & 99.79 \\
\hline 5 & 472 & 471 & 99.79 \\
\hline 6 & 270 & 269 & 99.63 \\
\hline 7 & 287 & 286 & 99.65 \\
\hline 8 & 302 & 302 & 100 \\
\hline 9 & 335 & 334 & 99.7 \\
\hline Total & 5400 & 5394 & 99.89 \\
\hline
\end{tabular}


Table 5: Confusion matrix with $\mathrm{k}=1$ (Zoning2)

\begin{tabular}{|c|c|c|c|c|c|c|c|c|c|c|}
\hline & 0 & 1 & 2 & 3 & 4 & 5 & 6 & 7 & 8 & 9 \\
\hline 0 & 1225 & 4 & 0 & 0 & 0 & 1 & 16 & 1 & 4 & 0 \\
\hline 1 & 0 & 961 & 3 & 115 & 1 & 1 & 2 & 6 & 0 & 0 \\
\hline 2 & 0 & 1 & 399 & 11 & 0 & 3 & 0 & 2 & 2 & 8 \\
\hline 3 & 0 & 0 & 6 & 471 & 0 & 3 & 0 & 15 & 0 & 1 \\
\hline 4 & 0 & 1 & 1 & 0 & 466 & 0 & 0 & 0 & 1 & 3 \\
\hline 5 & 0 & 1 & 0 & 3 & 1 & 456 & 2 & 0 & 2 & 7 \\
\hline 6 & 0 & 2 & 0 & 0 & 3 & 3 & 262 & 0 & 0 & 0 \\
\hline 7 & 0 & 0 & 0 & 3 & 0 & 0 & 0 & 278 & 0 & 6 \\
\hline 8 & 0 & 0 & 2 & 4 & 1 & 4 & 0 & 0 & 289 & 2 \\
\hline 9 & 1 & 0 & 1 & 1 & 4 & 0 & 1 & 3 & 3 & 321 \\
\hline
\end{tabular}

Table 6: Digit wise recognition accuracy with $k=1$ (Zoning2)

\begin{tabular}{|r|r|r|r|}
\hline Digit & Total & Correct & Percentage \\
\hline 0 & 1251 & 1225 & 97.92 \\
\hline 1 & 1089 & 961 & 88.25 \\
\hline 2 & 426 & 399 & 93.66 \\
\hline 3 & 496 & 471 & 94.96 \\
\hline 4 & 472 & 466 & 98.73 \\
\hline 5 & 472 & 456 & 96.61 \\
\hline 6 & 270 & 262 & 97.04 \\
\hline 7 & 287 & 278 & 96.86 \\
\hline 8 & 302 & 289 & 95.7 \\
\hline 9 & 335 & 321 & 95.82 \\
\hline Total & 5400 & 5128 & 94.96 \\
\hline
\end{tabular}

Table 7: Confusion matrix with $\mathrm{k}=1$ (Zoning3)

\begin{tabular}{|c|c|c|c|c|c|c|c|c|c|c|}
\hline & 0 & 1 & 2 & 3 & 4 & 5 & 6 & 7 & 8 & 9 \\
\hline 0 & 1223 & 2 & 0 & 9 & 1 & 0 & 13 & 0 & 2 & 1 \\
\hline 1 & 0 & 1082 & 1 & 0 & 1 & 0 & 3 & 1 & 1 & 0 \\
\hline 2 & 0 & 3 & 405 & 4 & 0 & 0 & 0 & 6 & 3 & 5 \\
\hline 3 & 0 & 0 & 7 & 461 & 0 & 12 & 0 & 4 & 11 & 1 \\
\hline 4 & 0 & 0 & 1 & 0 & 465 & 2 & 0 & 2 & 0 & 2 \\
\hline 5 & 0 & 2 & 5 & 12 & 2 & 445 & 0 & 0 & 6 & 0 \\
\hline 6 & 8 & 2 & 1 & 0 & 6 & 4 & 245 & 0 & 3 & 1 \\
\hline 7 & 0 & 1 & 1 & 13 & 0 & 1 & 0 & 266 & 1 & 4 \\
\hline 8 & 3 & 0 & 11 & 28 & 3 & 4 & 2 & 0 & 248 & 3 \\
\hline 9 & 0 & 2 & 1 & 4 & 3 & 0 & 1 & 10 & 1 & 313 \\
\hline
\end{tabular}

Table 8: Digit wise recognition accuracy with $k=1$ (Zoning3)

\begin{tabular}{|r|r|r|r|}
\hline Digit & Total & Correct & Percentage \\
\hline 0 & 1251 & 1223 & 97.76 \\
\hline 1 & 1089 & 1082 & 99.36 \\
\hline 2 & 426 & 405 & 95.07 \\
\hline 3 & 496 & 461 & 92.94 \\
\hline 4 & 472 & 465 & 98.52 \\
\hline 5 & 472 & 445 & 94.28 \\
\hline 6 & 270 & 245 & 90.74 \\
\hline 7 & 287 & 266 & 92.68 \\
\hline 8 & 302 & 248 & 82.12 \\
\hline 9 & 335 & 313 & 93.43 \\
\hline Total & 5400 & 5153 & 95.43 \\
\hline
\end{tabular}

Table 9: Confusion matrix with $\mathrm{k}=1$ (Zoning All)

\begin{tabular}{|c|c|c|c|c|c|c|c|c|c|c|}
\hline & 0 & 1 & 2 & 3 & 4 & 5 & 6 & 7 & 8 & 9 \\
\hline 0 & 1225 & 1 & 0 & 9 & 1 & 0 & 13 & 0 & 2 & 0 \\
\hline 1 & 0 & 971 & 1 & 112 & 1 & 0 & 3 & 0 & 1 & 0 \\
\hline 2 & 0 & 3 & 408 & 3 & 0 & 0 & 0 & 6 & 3 & 3 \\
\hline 3 & 0 & 0 & 8 & 461 & 0 & 11 & 0 & 4 & 11 & 1 \\
\hline 4 & 0 & 0 & 1 & 0 & 465 & 2 & 0 & 2 & 0 & 2 \\
\hline 5 & 0 & 1 & 4 & 12 & 2 & 447 & 0 & 0 & 6 & 0 \\
\hline 6 & 7 & 2 & 1 & 1 & 6 & 4 & 246 & 0 & 3 & 0 \\
\hline 7 & 0 & 0 & 1 & 14 & 0 & 1 & 0 & 266 & 1 & 4 \\
\hline 8 & 3 & 0 & 11 & 29 & 3 & 4 & 2 & 0 & 247 & 3 \\
\hline 9 & 0 & 2 & 1 & 4 & 3 & 0 & 1 & 10 & 1 & 313 \\
\hline
\end{tabular}

Table 10: Digit wise recognition accuracy with $k=1$ (Zoning3)

\begin{tabular}{|r|r|r|r|}
\hline Digit & Total & Correct & Percentage \\
\hline 0 & 1251 & 1225 & 97.92 \\
\hline 1 & 1089 & 971 & 89.16 \\
\hline 2 & 426 & 408 & 95.77 \\
\hline 3 & 496 & 461 & 92.94 \\
\hline 4 & 472 & 465 & 98.52 \\
\hline 5 & 472 & 447 & 94.7 \\
\hline 6 & 270 & 246 & 91.11 \\
\hline 7 & 287 & 266 & 92.68 \\
\hline 8 & 302 & 247 & 81.79 \\
\hline 9 & 335 & 313 & 93.43 \\
\hline Total & 5400 & 5049 & 93.5 \\
\hline
\end{tabular}

Table 11: Performance Analysis of All Zoning Techniques \begin{tabular}{|l|l}
\hline kNN Classifier & ZONING TECHNIQUES
\end{tabular}

\begin{tabular}{|c|c|c|c|c|}
\hline K & Zone1 & Zone2 & Zone3 & Zone All \\
\hline 1 & $99.89 \%$ & $94.96 \%$ & $95.43 \%$ & $93.50 \%$ \\
\hline 3 & $92.93 \%$ & $89.74 \%$ & $91.15 \%$ & $89.24 \%$ \\
\hline 5 & $86.37 \%$ & $76.08 \%$ & $83.87 \%$ & $84.22 \%$ \\
\hline
\end{tabular}

In this paper we have used Zoning Feature Extraction technique for extracting features from isolated numeric images with $\mathrm{k}-\mathrm{Ne}$ arest Neighbor classifier with the value of $\mathrm{k}=1$. This provides the best recognition accuracy rate among all the three zoning feature extraction techniques, which are defined in this paper.

Table 12: Performance Analysis of Zoning1 Techniques

\begin{tabular}{|c|c|c|c|c|}
\hline $\begin{array}{c}\text { Feature } \\
\text { Extraction } \\
\text { Method }\end{array}$ & Classifier & $\mathbf{k}$ & $\begin{array}{c}\text { No. of } \\
\text { Features }\end{array}$ & $\begin{array}{c}\text { Recognition } \\
\text { Accuracy }\end{array}$ \\
\hline Zoning 1 & $\begin{array}{c}\text { k-Nearest } \\
\text { neighbor }\end{array}$ & 1 & 36 & $99.89 \%$ \\
\hline
\end{tabular}

Here in the given table as we can see that by using different zoning feature extraction methods with $\mathrm{kNN}$ classifier which have different values of $k$, different recognition rates can be achieved. But with zoning1 feature extraction method with the value of $\mathrm{k}=1$ higher recognition rate is achieved, which is comparatively good than other recognition rates. So zoning1 feature extraction method with $\mathrm{kNN}$ classifier where value of $\mathrm{k}=1$ is adopted 


\section{CONCLUSION}

In this paper we have proposed Zone feature extraction method for the recognition of handwritten isolated numerals. Nearest neighbor classifier is used for classification and recognition. The recognition rate of $99.89 \%$ is achieved for handwritten isolated numerals. Our future work aims to improve classifier to achieve still better recognition rate and also to develop new zone based feature extractions algorithms, which provides efficient results.

\section{REFERENCES}

[1] P. Zhang, T. D. Bui, C. Y. Suen, "Hybrid Feature Extraction and Feature Selection for Improving Recognition Accuracy of Handwritten Numerals", Eight International Conference on Document Analysis and Recognition, pp.1520-1525,2005.

[2] M. Ziaratban, K. Faez, F. Faradji, "Language-Based Feature Extraction Using Template-Matching In Farsi/Arabic Handwritten Numeral Recognition", Ninth International Conference on Document Analysis and Recognition, pp. 297 - 301, 2007.

[3] J .Hu and H.Yan, "Structural Decomposition and Description of Printed and Handwritten Characters", proceedings of International Conference on Pattern Recognition, pp.230-234, Vienna, Austria, 1996.

[4] Heutte, L.; Moreau, J.V.; Paquet, T.; Lecourtier, Y.; Olivier, C "Combining structural and statistical features for the recognition of handwritten characters", Proceedings of the 13th International Conference on Pattern Recognition, vol.2, pp. 210 - 214, 1996.

[5] S.V. Rajashekararadhya, P. Vanaja Ranjan, "Zone based Feature Extraction Algorithm for Handwritten Numeral Recognition of Kannada Script", IEEE International Advance Computing Conference, pp. 525-528, 2009.

[6] Y. Hamamoto, S. Uchimura, M. Watanabe, T. Yasuda, and S. Tomita, "Recognition of Handwritten Numerals Using Gabor Features", Proceedings International Conference on Pattern Recognition, pp. 250 - 253, 1996.

[7] M. Watanabe, Y. Hamamoto, T. Yasuda, and S. Tomita, "Normalization Techniques of Handwritten Numerals for Gabor Filters", Computers, IEEE Transactions, pp. 303 307, 1997.
[8] Liu, C.-L.; Koga, M.; Fujisawa, H.; "Gabor feature extraction for character recognition: comparison with gradient feature", proceedings. Eighth International Conference on Document Analysis and Recognition, pp. $121-125,2005$.

[9] U. Pal, T. Wakabayashi and F. Kimura, "Handwritten Bangla Compound Character Recognition using Gradient Feature", 10th International Conference on Information Technology, pp. 208-213, 2007.

[10] Il-Seok Oh, C.Y Suen, "A Feature for character recognition based on Directional Distance Distributions", proceedings of the fourth conference on document analysis and recognition, pp. 288-292, 1997.

[11] M. Steuer, P. Caleb-Solly, Jim E. Smith., “An alternative approach for the evaluation of the neocognitron", Proceedings of ESANN, pp.125-130, 2001.

[12] D.S. Yeung, Hing-Yip Chan, Yau Chong Lau, "A neocognitron synthesized by production rule for handwritten character recognition", Proceedings, First International Symposium on Intelligence in Neural and Biological Systems, pp. 203 - 208, 1995.

[13] Kunihiko Fukushima, "Neocognitron of a New Version: Handwritten Digit Recognition", Proceedings, International Joint Conference on Neural Networks, pp. 1498-1503, 2001.

[14] Pramod Kumar Sharma , " Multiple Classifiers for Unconstrained Offline Handwritten Numeral Recognition", International Conference on Computational Intelligence and Multimedia Applications, pp. 344-348, 2007.

[15] Tian Ming, Zhuang Yi, and Chen Songcan, "Improving Support Vector Machine Classifier by Combining it with $\mathrm{k}$ Nearest Neighbor Principle Based on the Best Distance Measurement", Proceedings. IEEE in intelligent Transport System, pp. 373-378, 2003.

[16] S. V. Rajashekararadhya, P. VanajaRanjan , "Support Vector Machine based Handwritten Numeral Recognition of Kannada Script", Proceedings International Advance Computing Conference, pp. 381-386, 2009.

[17] Christopher J.C. Burges, "A tutorial on Support Vector Machines for Pattern Recognition", Data Mining and Knowledge Discovery, pp. 121-167, 1998. 